\title{
Análisis comparativo de la eficiencia estabilizadora de asfaltenos del aceite de cáscara de Anacardium occidentale y productos comerciales
}

\section{(Comparative analysis of the asphaltene stabilizing efficiency of Anacardium occidentale peel oil and commercial products)}

Dany Day Josefina Arriojas Tocuyo, Tomás Darío Marín Velásquez²

\begin{abstract}
Resumen
La investigación tuvo como objetivo comparar la eficiencia inhibidora y estabilizante de asfaltenos, en mezclas de aceite de cáscaras de Anacardium occidentale (CNSL) en diésel y productos dispersantes comerciales, para evaluar el aceite como alternativa de tratamiento químico antiasfalténico del petróleo crudo. Se determinó el umbral de floculación por precipitación con n-heptano y el punto de dispersión por adición de xileno, ambos mediante la observación directa de la formación de flóculos de asfaltenos en un microscopio óptico. El diseño experimental utilizado fue factorial, con cuatro variables respuestas (umbral de floculación, actividad inhibidora, índice de inestabilidad y eficiencia de estabilización) y dos factores experimentales (producto y dosis). Se evaluaron cinco productos con CNSL y tres productos comerciales, todos en dosis 2, 4, 6 y $8 \mu \mathrm{l}$ en $10 \mathrm{ml}$ de una muestra de petróleo crudo mediano. El análisis estadístico se basó en Anova multifactorial y test de diferencia mínima significativa de Fisher (LSD) con $\alpha=0.05$. Se obtuvo que los dos factores experimentales influyeron significativamente en las variables respuestas de forma individual y también sus interacciones. Los productos con CNSL fueron más eficientes como inhibidores y los comerciales más eficientes como estabilizadores.
\end{abstract}

Palabras clave

Anacardium occidentale; petróleo crudo; asfaltenos; inhibidor; floculación.

\begin{abstract}
The research objective was to compare the inhibiting and stabilizing efficiency of asphaltenes from Anacardium occidentale (CNSL) husk oil mixtures in diesel and commercial dispersant products, to evaluate the oil as an alternative anti-asphaltenes chemical treatment of crude oil. The precipitation flocculation onset with n-heptane and the dispersion point by addition of xylene were determined, both by direct observation of asphaltenes floc formation under an optical microscope. The experimental design used was factorial, with four response variables (flocculation onset, inhibitory activity, instability index and stabilization efficiency) and two experimental factors (product and dose). Five products with CNSL and three commercial products, all in doses 2, 4, 6 and $8 \mu$ in $10 \mathrm{ml}$ of a medium crude oil sample, were evaluated. The statistical analysis was based on multifactorial ANOVA and Fisher's minimal significant difference test (LSD) with $\alpha=0.05$. It was obtained that the two experimental factors significantly influenced the individual response variables and also their interactions. The products with CNSL were more efficient as inhibitors and the commercial ones more efficient as stabilizers.
\end{abstract}

\section{Keywords}

Anacardium occidentale; crude oil; asphaltenes; inhibitor; flocculation.

\section{Introducción}

Los asfaltenos son compuestos constituyentes del petróleo crudo, que están definidos químicamente como macromoléculas y contienen núcleos aromáticos y nafténicos, cadenas alifáticas y heteroátomos como el oxígeno, el nitrógeno o el azufre (Silva, Guimarães, Seidl, García, 
2013). También son definidos como estructuras o moléculas poliaromáticas altamente condensadas, compuestas principalmente de carbono, hidrógeno y una proporción menor de hetero elementos como: azufre, nitrógeno y oxígeno (Alrashidi y Nasr-El-Din, 2017). Los asfaltenos por lo general, han sido definidos por su solubilidad, como la fracción insoluble en hidrocarburos parafínicos lineales de bajo peso molecular, tales como n-heptano, pero soluble en compuestos aromáticos, como el tolueno (Guzmán, Ancheyta, Trejo y Rodríguez, 2017).

Los asfaltenos son considerados como compuestos polares, lo que es causado por los electrones de átomos como el azufre, el oxígeno y el nitrógeno (Hasanvand et al., 2018). Se ha determinado que, incluso en bajas concentraciones, la molécula de asfaltenos presenta tendencia a agregarse y precipitarse, lo que causa grandes daños en las diferentes etapas del procesamiento del petróleo, como consecuencia de obstrucciones ocasionadas por los depósitos orgánicos que producen (Guzmán et al., 2017; Hasanvand et al., 2018). La formación de depósitos de asfaltenos es un problema de importancia en la producción de petróleo, porque el depósito de asfaltenos perjudica la recuperación y la producción, al producir reducción de la permeabilidad de la roca del yacimiento y disminución del diámetro efectivo de los punzonados en la tubería de producción, lo que restringe el flujo de petróleo (Martins, Martins y Santos, 2018).

La estabilidad de los asfaltenos dentro del petróleo crudo, está definida como la capacidad de los mismos de mantenerse en suspensión coloidal, estabilizados por una capa de resina, en un sistema polidisperso denominado micela (Ashoori, Sharifi, Masoumi y Salehi, 2017; Li et al., 2020). Por lo tanto, para evitar la precipitación de asfaltenos y las subsecuentes consecuencias derivadas de este fenómeno, se utilizan, en las operaciones de tratamiento del petróleo crudo, productos químicos, denominados inhibidores o dispersantes de asfaltenos, cuya función es mantener a los asfaltenos estables, evitando la formación de agregados (Delgado, 2015).

El uso de dispersantes de asfaltenos, genera costos importantes a la industria petrolera, no solo de dinero, sino ambientales, debido a que los mismos están compuestos por compuestos activos y solventes como el tolueno o xileno, que son tóxicos y potenciales contaminantes de suelos y aguas (Alrashidi y Nasr-El-Din, 2017). Debido a lo anterior, se ha investigado el uso de compuestos químicos alternativos, como alcoholes de cadena corta (Martins et al., 2018), metil esteres, terpenos y etil lactato (Elochukwu, Saaid y Pilus, 2014). De igual forma, se han estudiado productos naturales, en particular, aceites vegetales y derivados de ellos como dispersantes de asfaltenos, entre los que se pueden citar, ácidos grasos como el láurico, palmítico y oleico (Alrashidi, Afra y Nasr-El-Din, 2019), los aceites de coco y de andiroba (Alrashidi y Nasr-El-Din, 2017; Marín, 2019), los aceites de nabo, almendra amarga, sésamo, manzanilla, jengibre, avellana, oliva y romero (Mardani, Mokhtari y Soltani, 2018), aceite de Jatropha curcas (Marín, Marcano y Febres, 2016).

El Anacardium occidentales, conocido como marañón, merey, nuez de la india, cajú o anacardo, es un árbol originario de la América tropical, que se distribuye desde el sur de la Florida en Estados Unidos, hasta Brasil (Taiwo, 2015). El aceite de la cáscara de la semilla de A. occidentale, también conocido como Cashew Nut Shell Liquid (CNSL) se extrae mediante disolventes y su composición principal aproximada es entre 71-82\% de ácido anacárdico, 1.6 a 9.2\% de Cardanol y 13.8 a $20.3 \%$ de Cardol (Nunes et al., 2019) y ha sido utilizado en diferentes investigaciones y en campos diferentes, como antibacterial (Kanehashi et al., 2015) y como antioxidante aplicado a biocombustible (Sánchez, Chávez, Ríos y Cardona, 2015).

Debido a que otros aceites vegetales han demostrado ser potenciales alternativas para el tratamiento de asfaltenos, y tomando en cuenta que el $A$. occidentale es una planta abundante en buena parte de América y que en algunos casos como en Brasil o Venezuela, crece de manera sil- 
vestre, se planteó la evaluación del CNSL (como estabilizador de asfaltenos), en comparación con productos comerciales utilizados como dispersantes, a través de un proceso experimental a nivel de laboratorio, y con la finalidad de estudiar si el mismo es una potencial alternativa a dichos productos.

\section{Metodología}

\subsection{Caracterización de la muestra de petróleo crudo}

La muestra de petróleo crudo utilizada se obtuvo de un pozo productor del Campo El Furrial, ubicado en la población del mismo nombre en el Estado Monagas, Venezuela. La misma fue caracterizada para obtener ciertas propiedades básicas como gravedad API, mediante la norma estándar ASTM D287 (2012); viscosidad dinámica por la norma ASTM D2196 (2018), porcentaje de agua y sedimentos (\% AyS), a través de la norma ASTM D4007 (2016), y su composición SARA aplicando el procedimiento descrito en la norma ASTM D2007 (2011), a partir de la cual se determinó el índice de estabilidad coloidal mediante la ecuación 1 (Guzmán et al., 2017).

$$
C I I=\frac{(\% \text { Saturados }+\% \text { Asfaltenos })}{(\% \text { Aromáticos }+\% \text { Resinas })}
$$

También se determinó su umbral de floculación de asfaltenos (UF) mediante el principio de titulación con n-heptano (Oliensis Spot Test Number) modificado mediante microscopía óptica (Abrahamsen, 2012; Kraiwattanawong et al., 2009; Marín, 2019). En el procedimiento para determinar el UF se aplicó n-heptano en proporciones sucesivas de $0.5 \mathrm{ml}$ a una muestra de $10 \mathrm{ml}$ de petróleo crudo sometido a agitación magnética y a temperatura constante de $25^{\circ} \mathrm{C}$. Luego de agitar por 5 min, por cada aplicación se extrajo una alícuota del petróleo crudo y se observó en un microscopio óptico con ocular 10X para constatar la formación de los flóculos de asfaltenos. Al observar la formación de los flóculos, se anotó el volumen total de n-heptano (ml) aplicado, como el UF de los asfaltenos, en caso de no observarse flóculos, se repite el proceso desde la adición de $0.5 \mathrm{ml}$ más de n-heptano, hasta obtener el UF. El esquema se muestra en la figura 1.

Figura 1. Esquema el ensayo para determinar el UF

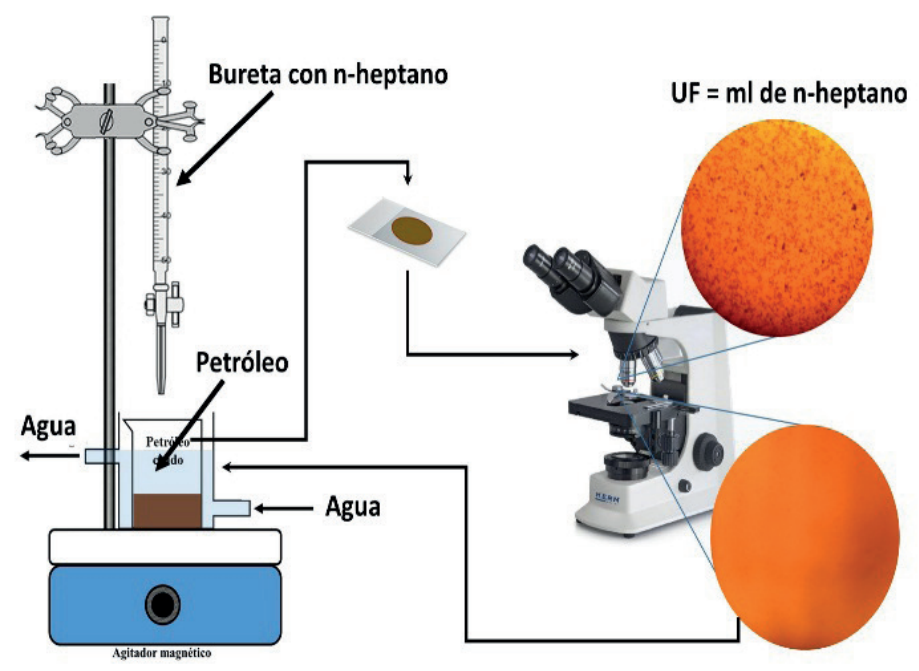

Fuente: elaboración propia. 
También se determinó el punto de dispersión de los asfaltenos (PD) mediante aplicación de xileno a la muestra de petróleo, luego de obtener el UF, en aplicaciones sucesivas de $0.1 \mathrm{ml}$, hasta dejar de observar los agregados de asfaltenos en el microscopio (Marín, 2019).

Con los valores de UF y PD se determinó el índice de inestabilidad de asfaltenos (INE), como la relación entre ambos parámetros, tal como se muestra en la ecuación 2.

$$
I N E=\frac{P D}{U F}
$$

El INE indica que mientras menor sea la relación entre PD y UF, mayor será la estabilidad de los asfaltenos en el petróleo crudo, pues se necesita menor cantidad de solvente (xileno) para dispersar las partículas de asfaltenos agregadas mediante n-heptano (Marín et al., 2016).

\subsection{Obtención del aceite de cáscaras de semillas de A. occidentale (CNSL)}

Las cáscaras de A. occidentale fueron obtenidas de frutos recolectados en la población de El Furrial y poblaciones vecinas (Musipán y Punta de Mata), directamente de árboles sembrados como soportes en cercos de fincas ganaderas. Se escogieron cáscaras de pseudofrutos maduros y se separaron manualmente de la nuez, se trituraron igualmente de forma manual y se secaron en estufa de laboratorio a una temperatura de $80^{\circ} \mathrm{C}$ para eliminar el exceso de humedad.

Para la extracción del aceite se utilizó un equipo Sohxlet de $500 \mathrm{ml}$ de capacidad y como solvente n-hexano grado analítico. La extracción se realizó con una proporción de $300 \mathrm{ml}$ de solvente por $30 \mathrm{~g}$ de cáscara triturada y seca. El proceso se realizó durante cuatro horas (Kusrini et al., 2018; Riss et al., 2015). El extracto se concentró en un rotoevaporador y se almacenó a condiciones de laboratorio $\left(25^{\circ} \mathrm{C}\right)$.

\subsection{Preparación de productos a evaluar}

Se prepararon tres productos con tres concentraciones de CNSL como componente activo y combustible diésel como solvente (40, 60 y $80 \%$ CNSL), para evaluar el efecto de la concentración del aceite sobre el UF de asfaltenos. Las mezclas y los tres productos comerciales fueron aplicadas al petróleo crudo en cuatro dosis $(2,4,6$ y $8 \mu \mathrm{l}$ por $10 \mathrm{ml}$ de petróleo). Adicionalmente se aplicó diésel puro y CNSL puro en las mismas dosis. Luego de aplicar el producto al petróleo crudo, se procedió a determinar el UF de los asfaltenos y se comparó con el UF original, con lo cual se obtuvo la actividad inhibidora relativa porcentual (ARP), mediante la ecuación 3.

$$
A R P=\frac{U F_{\text {dosificado }}-U F_{\text {Original }}}{U F_{\text {Original }}} \times 100
$$

Además, para cada muestra de petróleo crudo dosificada con los productos evaluados se determinó el PD y se calculó el INE mediante la ecuación 2, y se determinó la eficiencia de estabilización (EF) mediante la aplicación de la ecuación 4.

$$
E F=\frac{I N E_{\text {original }}-I N E_{\text {Dosificado }}}{I N E_{\text {Original }}} \times 100
$$




\subsection{Diseño experimental}

Se estableció un diseño experimental factorial con cuatro variables respuesta (UF, ARP, INE, EF) y 2 factores experimentales (producto y dosis). El diseño seleccionado tiene 96 ejecuciones, con una muestra para tomada en cada ejecución. El modelo por defecto es de interacciones, de 2 factores con 34 coeficientes. Los factores experimentales se describen en la Tabla 1.

Tabla 1. Descripción de los factores experimentales

\begin{tabular}{|c|l|l|}
\hline Factor & Nomenclatura & \multicolumn{1}{|c|}{ Descripción } \\
\hline \multirow{5}{*}{ Producto } & P0 & $100 \%$ Diésel \\
\cline { 2 - 3 } & P1 & $40 \%$ CNSL \\
\cline { 2 - 3 } & P2 & $60 \%$ CNSL \\
\cline { 2 - 3 } & P3 & $80 \%$ CNSL \\
\cline { 2 - 3 } & P4 & $100 \%$ CNSL \\
\cline { 2 - 3 } & PC1 & Producto comercial 1 \\
\cline { 2 - 3 } & PC2 & Producto comercial 2 \\
\cline { 2 - 3 } & PC3 & Producto comercial 3 \\
\hline \multirow{5}{*}{ Dosis } & D2 & $4 \mu \mathrm{l}$ de producto \\
\cline { 2 - 3 } & D4 & $6 \mu$ de producto producto \\
\cline { 2 - 3 } & D6 & $8 \mu \mathrm{l}$ de producto \\
\cline { 2 - 3 } & D8 & \\
\hline
\end{tabular}

Fuente: elaboración propia

\subsection{Tratamiento estadístico de los datos}

Para elaborar el diseño estadístico planteado se utilizó el paquete estadístico Statgraphics Centurion XVII. Se aplicó Anova multifactorial y test de rangos múltiples, basado en la diferencia mínima significativa de Fisher (Lowest Significant Difference - LSD) con un nivel de confianza de $95 \%(\alpha=0.05)$.

\section{Resultados y discusión}

\subsection{Características de la muestra de petróleo}

Las características medidas mediante los ensayos de laboratorio, de la muestra de petróleo crudo utilizada, se muestran en la tabla 2.

El valor de la gravedad API del petróleo lo clasifica como mediano o medio (API entre 22 y 32). El porcentaje de agua y sedimentos de 0.1, indica que el petróleo contiene poca agua, es decir es un petróleo seco o limpio. El valor de API determinado coincide con el rango reportado de 20.6 a 29.4 para petróleos de diferentes pozos del Campo el Furrial (Guevara et al., 2018). El \% AyS al igual que la API, varía de un pozo a otro y es función del método de producción aplicado; sin embargo el valor obtenido de $0.1 \%$, es consistente con los reportados para 6 pozos del Campo El Furrial, de donde proviene la muestra (Rodríguez, 2011). En cuanto a la composición SARA, se observa una mayor cantidad de compuestos saturados y aromáticos (71.7\%). La composición SARA de mezclas de petróleos del campo El Furrial para el año 2015 fue reportada por Guevara et al. (2018) en 63 \% de saturados 
y aromáticos, el cual es un valor menor al obtenido en la presente investigación. Sin embargo, se debe tomar en cuenta que la composición del petróleo varía incluso entre pozos del mismo campo y también lo hace con el tiempo, por lo que no se puede establecer una comparación y lo obtenido es representativo de la muestra utilizada. Igualmente se observó una diferencia importante entre los porcentajes de resinas y asfaltenos obtenidos, respecto a los reportados por Guevara et al. (2018), los cuales fueron de 25 y 7 \% respectivamente, lo que por supuesto se debe a la complejidad composicional del petróleo, que fue mencionada anteriormente.

Tabla 2. Propiedades de la muestra de petróleo crudo

\begin{tabular}{|l|l|l|}
\hline \multicolumn{1}{|c|}{ Propiedad } & \multicolumn{1}{c|}{ Valor } & \multicolumn{1}{c|}{ Unidad } \\
\hline Gravedad API & 27.4 & ${ }^{\circ} \mathrm{API}$ \\
\hline Viscosidad a $25^{\circ} \mathrm{C}$ & 17.7 & $\mathrm{CP}$ \\
\hline$\%$ AyS & 0.1 & $\%$ \\
\hline \% Saturados & 50.5 & $\%$ \\
\hline \% Aromáticos & 21.2 & $\%$ \\
\hline \% Resinas & 5.1 & $\%$ \\
\hline \% Asfaltenos & 3.1 & $\%$ \\
\hline \% Volátiles & 20.1 & $\%$ \\
\hline UF & $7.8 \pm 0.3$ & $\mathrm{mlC7}$ \\
\hline PD & $4.0 \pm 0.2$ & $\mathrm{mlXileno}$ \\
\hline INE & 0.51 & $\mathrm{mlXileno} / \mathrm{mlC7}$ \\
\hline
\end{tabular}

Fuente: elaboración propia.

El valor de Cll calculado a través de la ecuación 1 dio como resultado 2.03, lo que caracteriza a la muestra como un petróleo crudo inestable $(C \| \geq 0.9)$, es decir, tiene tendencia a producir precipitación de asfaltenos (Guzmán et al., 2017). Lo anterior es consistente con lo obtenido por Guevara et al. (2018) para tres muestras de petróleo crudo pertenecientes también al campo el Furrial, las cuales mostraron valores de Cll entre 3.6 y 4.1, por lo que en todos los casos se observan petróleos inestables. El UF indica que se necesitan $7.8 \pm 0.3 \mathrm{ml}$ de n-heptano por cada $10 \mathrm{ml}$ del petróleo crudo, para iniciar la formación de agregados de asfaltenos. El valor de PD de $4.0 \pm 0.2$ indica que se necesitaron $4 \mathrm{ml}$ de xileno para dispersar los agregados formados en el UF. El INE de $0.51 \mathrm{ml}$ de xileno por $\mathrm{ml}$ de $\mathrm{n}$-heptano, y fue menor al valor de 1.4 reportado por Marín (2019) para una muestra de 30.8 API del mismo campo petrolero. El valor de INE no puede ser comparado de manera estricta, debido a que es propio de cada petróleo crudo, por lo que aun cuando se tengan muestras del mismo campo, sus composiciones pueden ser diferentes y por ende sus INE pueden ser diferentes.

\subsection{Resultados de las variables medidas}

Los valores obtenidos para cada una de las variables medidas (UF, ARP, INE, EF), se muestran en la tabla 3. Se observa que el producto P0 el cual es 100 \% diésel no ejerció un efecto importante, ya que sus UF fueron similares a los originales, por lo que se comprueba que el mismo no produce ningún cambio en la estabilidad de los asfaltenos, lo que se observa también en los valores de ARP e INE de 0.43 y $3.92 \%$ respectivamente. Los productos que contienen CNSL, arrojaron 
valores de UF en general mayores al UF original, así mismo los INE fueron menores al original, lo que indicó que ejercen actividad inhibidora y estabilizante de los asfaltenos (Zeng et al., 2019). La mayor actividad inhibidora para los productos con CNSL, se obtuvo con el producto P3 a la dosis de $4 \mu \mathrm{l}$ con $43.16 \%$ y la mayor eficiencia estabilizadora se calculó para el producto P4 a la dosis de $8 \mu$ le $75.16 \%$. Los productos comerciales, también mostraron actividad inhibidora en una ARP mayor para PC3 a la dosis de $4 \mu$ con $45.30 \%$ y eficiencia de estabilización máxima de $93.46 \%$ para PC1 a la dosis de $8 \mu \mathrm{l}$. El comportamiento de cada producto depende de su composición (Lim et al., 2020), en el caso específico los productos con CNSL, difieren respecto a la concentración, lo que influye en su ARP y EF, de igual forma, los productos comerciales, aunque su composición no es aportada por las casas comerciales que las venden, también tiene diferentes composiciones, lo que se comprueba con los resultados diferentes observados.

Tabla 3. Resultados obtenidos para las variables en estudio

\begin{tabular}{|c|c|c|c|c|c|}
\hline Producto & Dosis & UF & ARP & INE & EF \\
\hline \multirow{4}{*}{ PO } & 2 & 7.8 & 0.43 & 0.49 & 3.92 \\
\hline & 4 & 7.8 & 0.43 & 0.49 & 3.92 \\
\hline & 6 & 7.5 & 0.00 & 0.49 & 3.27 \\
\hline & 8 & 7.8 & 0.43 & 0.48 & 6.54 \\
\hline \multirow{4}{*}{ P1 } & 2 & 8.3 & 6.84 & 0.18 & 64.05 \\
\hline & 4 & 8.7 & 11.11 & 0.18 & 65.36 \\
\hline & 6 & 8.3 & 6.84 & 0.17 & 66.67 \\
\hline & 8 & 8.0 & 2.56 & 0.16 & 68.63 \\
\hline \multirow{4}{*}{ P2 } & 2 & 7.3 & 0.00 & 0.20 & 60.13 \\
\hline & 4 & 8.5 & 8.97 & 0.18 & 65.36 \\
\hline & 6 & 9.8 & 26.07 & 0.15 & 70.59 \\
\hline & 8 & 9.7 & 23.93 & 0.14 & 72.55 \\
\hline \multirow{4}{*}{ P3 } & 2 & 9.7 & 23.93 & 0.16 & 68.63 \\
\hline & 4 & 11.2 & 43.16 & 0.14 & 72.55 \\
\hline & 6 & 9.5 & 21.79 & 0.16 & 69.28 \\
\hline & 8 & 8.7 & 11.11 & 0.17 & 67.32 \\
\hline \multirow{4}{*}{ P4 } & 2 & 9.2 & 17.52 & 0.16 & 69.28 \\
\hline & 4 & 9.7 & 23.93 & 0.15 & 70.59 \\
\hline & 6 & 10.3 & 32.48 & 0.13 & 73.86 \\
\hline & 8 & 10.5 & 34.62 & 0.13 & 75.16 \\
\hline \multirow{4}{*}{ PC1 } & 2 & 8.3 & 6.84 & 0.09 & 83.01 \\
\hline & 4 & 8.7 & 11.11 & 0.07 & 86.93 \\
\hline & 6 & 7.5 & 0.00 & 0.05 & 90.85 \\
\hline & 8 & 7.0 & 0.00 & 0.03 & 93.46 \\
\hline \multirow{4}{*}{ PC2 } & 2 & 7.5 & 0.00 & 0.10 & 81.05 \\
\hline & 4 & 8.3 & 6.84 & 0.05 & 90.85 \\
\hline & 6 & 10.0 & 28.21 & 0.07 & 86.27 \\
\hline & 8 & 9.3 & 19.66 & 0.07 & 86.27 \\
\hline \multirow{4}{*}{ PC3 } & 2 & 9.7 & 23.93 & 0.07 & 86.93 \\
\hline & 4 & 11.3 & 45.30 & 0.04 & 92.81 \\
\hline & 6 & 9.3 & 19.66 & 0.05 & 90.20 \\
\hline & 8 & 8.3 & 6.84 & 0.04 & 91.50 \\
\hline
\end{tabular}

Fuente: elaboración propia 
La eficiencia de inhibición obtenida es consistente con la reportada por Meléndez-Álvarez et al. (2016) para una muestra de petróleo crudo de Oriente Medio, que fue en promedio entre 40.4 y $54.1 \%$, aunque fue una muestra de diferente composición comparada con la utilizada. Igualmente muestras de petróleo crudo provenientes de Houston, TX, tratadas con inhibidores de asfaltenos mostraron porcentajes de eficiencia entre 42.0 y 67.0 \% (Kuang et al., 2019), lo que demuestra que a pesar de las diferencias de composición de las muestras, los resultados máximos obtenidos en la presente investigación están dentro de este rango. El aceite de coco reportó valores de eficiencia estabilizadora máxima de 78.60 \% en una muestra de petróleo del campo Carito, Monagas, Venezuela, que es similar al máximo obtenido para el CNSL que fue de $75.55 \%$ (Marín, 2019), la diferencia, como ya se ha mencionado, se debe a las diferencias en composición del petróleo y de los aceites vegetales utilizados. Bello, Manzano y Marín (2015) reportaron eficiencias para productos químicos dispersantes comerciales en muestras de petróleo crudo del campo El Furrial con rangos máximos entre 46.66 y $69.44 \%$, los cuales fueron menores a los rangos obtenidos con los productos utilizados, donde los productos formulados con CNSL tuvieron un rango entre 60.13 y $75.16 \%$ y para los productos comerciales entre 83.01 y $93.46 \%$.

\subsection{Resultados del análisis estadístico}

El análisis Anova multifactorial aplicado al diseño experimental planteado, arrojó los resultados que se muestran en la Tabla 4.

Tabla 4. Resultados del Anova multifactorial aplicado a las cuatro variables respuesta

\begin{tabular}{|l|l|l|l|l|}
\hline \multirow{2}{*}{ Factores } & \multicolumn{4}{c|}{ Valor-p } \\
\cline { 2 - 5 } & \multicolumn{1}{c|}{ UF } & \multicolumn{1}{c|}{ ARP } & \multicolumn{1}{c|}{ EF } \\
\hline \multicolumn{5}{|c|}{ Efectos } \\
\hline Bloque & 0.1133 & 0.1133 & 0.6267 & 0.6267 \\
\hline A: Producto & 0.0000 & 0.0000 & 0.0000 & 0.0000 \\
\hline B: Dosis & 0.0000 & 0.0000 & 0.0000 & 0.0000 \\
\hline \multicolumn{5}{|l|}{ Interacciones } \\
\hline AB & 0.0534 & 0.0534 & 0.5553 & 0.5553 \\
\hline AC & 0.3335 & 0.3335 & 0.8638 & 0.8638 \\
\hline BC & 0.0000 & 0.0000 & 0.0012 & 0.0012 \\
\hline
\end{tabular}

Fuente: elaboración propia a partir de resultados del Statgraphics Centurion XVII.

En la tabla 4 se observa que en el caso de los bloques experimentales (repeticiones) no se obtuvo diferencia significativa (Valor- $p>0.05$ ), lo que indica que los valores medios de las variables obtenidos entre las repeticiones fueron estadísticamente iguales, lo que da consistencia a los ensayos realizados. Los factores experimentales producto y dosis ejercieron influencia significativa sobre cada una de las variables (Valor-p < 0.05). En cuanto a las interacciones entre los factores, se observó que, interbloques (AB y AC) no fueron significativas, por lo que se puede decir que al comparar los resultados de las variables por cada repetición, estas fueron estadísticamente iguales. La interacción entre los factores (BC) sí fue significativa, por lo que no solo los factores influyen de manera individual sobre las variables, sino que también es influyente la interacción entre ellos (producto $x$ dosis). Al comparar con el resultado reportado por Marin et al. (2016) para el aceite de J. curcas, se observa un resultado que no coincide con el obtenido en la presente investigación, ya que para la J. curcas, el porcentaje de aceite en la mezcla (Produc- 
to) no influyó significativamente, solo se reportó influencia de la dosis. Lo anterior demuestra que los resultados pueden estar influidos por el tipo de aceite utilizado, además, como ya se ha mencionado, del tipo de petróleo crudo utilizado.

Para establecer el comportamiento individual del factor producto sobre las variables ARP y EF, se realizó el test LSD de Fisher, cuyos resultados se muestran en las tablas 5 y 6 . En la tabla 5 se observa que en cuanto a la eficiencia inhibidora, el PC1 no mostró diferencias significativas respecto al PO, lo que indica que este producto comercial se comporta de forma similar al diésel puro. El producto P1 mostró una eficiencia mayor a los anteriores, pero baja y diferente comparado con los otros productos. Los productos P2 y PC2 no mostraron diferencias significativas, lo que indica que su efecto sobre ARP fue estadísticamente igual, a pesar de que P2 tuvo una ARP promedio mayor.

Tabla 5. Prueba LSD de Fisher para la variable ARP

\begin{tabular}{|l|c|c|c|c|}
\hline \multicolumn{1}{|c|}{ Producto } & Casos & Media & Sigma & Grupos \\
\hline P0 & 12 & -0.64 & 1.56 & A \\
\hline PC1 & 12 & 0.96 & 1.56 & $\mathrm{~A}$ \\
\hline P1 & 12 & 6.84 & 1.56 & $\mathrm{~B}$ \\
\hline PC2 & 12 & 12.71 & 1.56 & $\mathrm{C}$ \\
\hline P2 & 12 & 13.25 & 1.56 & $\mathrm{D}$ \\
\hline PC3 & 12 & 23.93 & 1.56 & $\mathrm{D}$ \\
\hline P3 & 12 & 25.00 & 1.56 & $\mathrm{D}$ \\
\hline P4 & 12 & 27.14 & 1.56 & \\
\hline
\end{tabular}

Letras diferentes representan diferencias significativas con Valor- $p<0.05$ Fuente: elaboración propia.

Los productos con mayor ARP fueron los P3, P4 y PC3, que además no arrojaron diferencias significativas entre ellos y se observa cómo los productos con CNSL fueron los más eficientes. Se demostró, sobre la base de la eficiencia inhibidora, que los productos elaborados mezclando CNSL y diésel son posibles sustitutos de los productos químicos comerciales, sobre todo los que presentan mayores porcentajes del aceite, e incluso el aceite puro. En la evaluación de eficiencia dispersora de asfaltenos de diferentes productos aplicados a una muestra de $21^{\circ} \mathrm{API}$ del campo El Furrial, Pereira et al. (2011) reportaron ARP entre 2.0 y $73.5 \%$, por lo que se puede decir que los resultados obtenidos en la investigación coinciden con este rango y se destaca que las mayores eficiencias obtenidas son similares e incluso mayores al producto Dodecanol, que reportó 23.7 \%.

Tabla 6. Prueba LSD de Fisher para la variable EF

\begin{tabular}{|l|c|c|c|c|}
\hline Producto & Casos & Media & Sigma & Grupos Homogéneos \\
\hline P0 & 12 & 4.41 & 0.75 & $\mathrm{~A}$ \\
\hline P1 & 12 & 66.18 & 0.75 & $\mathrm{~B}$ \\
\hline P2 & 12 & 67.16 & 0.75 & $\mathrm{~B}$ \\
\hline P3 & 12 & 69.44 & 0.75 & $\mathrm{C}$ \\
\hline P4 & 12 & 72.22 & 0.75 & $\mathrm{D}$ \\
\hline PC1 & 12 & 86.11 & 0.75 & $\mathrm{~F}$ \\
\hline PC2 & 12 & 88.56 & 0.75 & $\mathrm{~F}$ \\
\hline PC3 & 12 & 90.36 & 0.75 & \\
\hline
\end{tabular}

Letras diferentes representan diferencias significativas con Valor- $p<0.05$

Fuente: elaboración propia. 
El comportamiento de los productos en función a EF fue diferente a lo observado con la ARP; los productos con CNSL presentaron eficiencias menores a los productos comerciales. El diésel puro (PO) tuvo eficiencia baja y con diferencia significativa comparado con los demás productos. Las eficiencias de los productos P1 y P2 no mostraron diferencias significativas, pero fueron diferentes respecto a los demás, el resto de los productos con CNSL tuvieron EF con diferencias entre ellos, lo que indica que al aumentar el CNSL en la mezcla de 80 a $100 \%$ la diferencia de EF fue significativa. De los productos comerciales, solo el PC1 tuvo diferencia significativa respecto a los otros dos, los cuales no presentaron diferencias, aun cuando PC3 fue el de mayor valor de EF con $90.36 \%$.

Al comparar la eficiencia de los productos evaluados con la eficiencia máxima reportada para un producto comercial, aplicado a un petróleo crudo ligero $\left(36.8^{\circ} \mathrm{API}\right)$ de Kuwait (Ghloum et al., 2019), se observó que la misma fue de 58.0 \% máxima, valor inferior a lo obtenido, incluso para el producto de menos eficiencia que fue el P1 (66.18\%). Las diferencias se deben a que el petróleo de Kuwait tiene características diferentes al utilizado en la investigación, sin embargo es un hecho el que los productos elaborados con base en CNSL mostraron eficiencias que pueden superar a la de productos comerciales. Así mismo, los productos con CNSL igualaron la eficiencia máxima reportada por Kuang et al. (2019) para productos aplicados a muestras de petróleos crudos mexicanos, lo cual fue de $67.0 \%$. Al comparar la eficiencia de los productos elaborados con CNSL con otros aceites vegetales, se observa que están dentro de los rangos reportados por Mardani et al. (2018) para los aceites de manzanilla (64 \%) y aceite de almendras dulces (82 \%). Así mismo, el aceite de J. curcas reportó una eficiencia máxima de 88.33\% (Marín et al., 2016) superando el máximo obtenido en esta investigación. De igual manera, el aceite de CNSL evaluado fue superado por el aceite de coco, que reportó eficiencias que llegaron hasta 93.75 \% (Bello et al., 2015). La comparación entre los productos formulados con CNSL y los productos químicos mostró que en relación a la eficiencia inhibidora (ARP), los productos con CNSL fueron superiores a los comerciales, no asi al comprar las eficiencias estabilizadores (EF) donde los valores mayores fueron los de los productos comerciales.

La comparación de los resultados con investigaciones precedentes es consistente con la observación de la importancia de la composición del petróleo crudo, sobre la eficiencia de los productos inhibidores/dispersantes de asfaltenos (Bello et al., 2015). Se ha investigado y establecido que el efecto de la composición está en función de la relación porcentual de los componentes SARA del petróleo y en especial la relación entre resinas y asfaltenos y entre saturados y asfaltenos (Gabrienko, Martyanov y Kazarian, 2015). Así mismo, la influencia de la composición del petróleo crudo sobre los inhibidores/dispersantes de asfaltenos se explicó, además, por la composición de los mismos asfaltenos y por el tipo de solvente utilizado para obtener el UF, es decir, los asfaltenos floculados por parafinas de menor peso molecular, son más estables que las fracciones floculadas por parafinas de mayor peso molecular (Pereira et al., 2011). En cuanto a la comparación de los costos del producto con CNSL y diésel con el de un dispersante comercial, según el portal Quiminet (2020), un litro de dispersante de asfaltenos tiene un costo promedio entre 2.3 y 3.0 US\$ por litro y en el portal Alibaba (2020) se menciona que el precio del CNSL es de aproximadamente 0.3 US\$ por litro, si se considera utilizarlo puro es evidente un ahorro entre 2.0 y 2.7 US\$ por litro de dispersante aplicado. 


\section{Conclusiones y recomendaciones}

Los productos elaborados al mezclar CNSL y diésel, presentan tanto actividad inhibidora como eficiencia de estabilización de asfaltenos, lo que indica que el aceite es potencial alternativa para el tratamiento de los asfaltenos en el petróleo crudo.

Al comparar la eficiencia inhibidora de los asfaltenos (ARP) de los productos evaluados, se concluye que las mezclas con 80 \% CNSL en diésel y el CNSL puro tienen un mejor comportamiento que los productos comerciales, al ser aplicados a la muestra de petróleo crudo estudiado y bajo las condiciones del diseño experimental aplicado.

A partir de la comparación de los productos elaborados con CNSL y los productos comerciales, basada en la eficiencia de estabilización (EF), se concluyó que los productos comerciales superan en eficiencia a los formulados con CNSL y Diésel; sin embargo, estos últimos arrojaron valores de EF que superan a las de otros productos evaluados en investigaciones precedentes, por lo que se puede considerar que los mismos presentan eficiencias estabilizantes aceptables al ser aplicadas a la muestra de crudo utilizada en la investigación.

Se recomienda continuar investigando sobre el uso del aceite de A. occidentale como alternativa para el tratamiento de los asfaltenos en el petróleo crudo a nivel de laboratorio, para establecer cómo influyen otras variables sobre su desempeño, tales como la temperatura, la presión y el tipo de petróleo crudo.

\section{Bibliografía}

Abrahamsen, E. L. (2012). Organic Flow Assurance: Asphaltene Dispersant/Inhibitor Formulation Development through Experimental Design. Stavanger: Schlumberger Limited.

Alibaba. (2020). Cashew Nut Shell Oil Price. Disponible en: http://bit.do/cashewoilprice

Alrashidi, H., y Nasr-El-Din, H. A. (2017). Evaluation of Eco Friendly Bio-Oil Dispersants on the Inhibition of Asphaltene Precipitation in a Kuwaiti Crude Oil. In the Abu Dhabi International Petroleum Exhibition \& Conference, SPE-188232-MS. Abu Dhabi. doi: 10.2118/188232-ms

Alrashidi, H.; Afra, S. y Nasr-El-Din, H. A. (2019). Application of Natural Fatty Acids as Asphaltenes Solvents with Inhibition and Dispersion Effects: A Mechanistic Study. Journal of Petroleum Science and Engineering, 172, 724-730. doi: 10.1016/j.petrol.2018.08.066

Ashoori, S.; Sharifi, M.; Masoumi, M. y Salehi, M. M. (2017). The Relationship between SARA Fractions and Crude Oil Stability. Egyptian Journal of Petroleum, 26, 209-213. doi: 10.1016/j.ejpe.2016.04.002

ASTM D287. (2012). Standard Test Method for API Gravity of Crude Petroleum and Petroleum Products (Hydrometer Method). USA: American Society of Testing Materials.

ASTM D2007. (2011). Standard Test Method for Characteristic Groups in Rubber Extender and Processing Oils and Other Petroleum-Derived Oils by the Clay-Gel Absorption Chromatographic Method. USA: American Society of Testing Materials.

ASTM D2196. (2018). Standard Test Methods for Rheological Properties of Non-Newtonian Materials by Rotational Viscometer. USA: American Society of Testing Materials.

ASTM D4007. (2016). Standard Test Method for Water and Sediment in Crude Oil by the Centrifuge Method (Laboratory Procedure). USA: American Society of Testing Materials.

Bello, Y. B.; Manzano, J. R. y Marín, T. D. (2015). Análisis comparativo de la eficiencia dispersora de asfaltenos de productos a base de aceite de coco (Cocus nucifera) como componente activo y dispersantes comerciales aplicados a muestras de petróleo del Campo el Furrial. Revista Tecnológica Espol-RTE, 28(2), 51-61. Estado Monagas, Venezuela.

Delgado, J.G. (2015). Precipitación de asfaltenos. Mérida, Venezuela: Universidad de los Andes. 
Elochukwu, O. H.; Saaid, I. M. y Pilus, R. M. (2014). Organic Deposit Remediation Using Environmentally Benign Solvents: A Review. ARPNJournal of Engineering and Applied Sciences, 9(10), 1930-1935.

Gabrienko, A. A.; Martyanov, O. N. y Kazarian, S. G. (2015). Effect of Temperature and Composition on the Stability of Crude Oil Blends Studied with Chemical Imaging In Situ. Energy \& Fuel, 29(11), 71147123. doi: 10.1021/acs.energyfuels.5b01880

Ghloum, E. F.; Rashed, A. M.; Safa, M. A.; Sablit, R. C. y Al-Jouhar, S. M. (2019). Mitigation of Asphaltenes Precipitation Phenomenon Via Chemical Inhibitors. Journal of Petroleum Science and Engineering, 175, 495-507. doi: 10.1016/j.petrol.2018.12.071

Guevara, S.; Parra, M.; Malavé, V.; Castillo, L. y Márquez, I. (2018). Efecto de la implementación del método de levantamiento artificial por gas sobre la composición del crudo del campo El Furrial. Revista Tecnológica Espol - RTE, 31(1), 1-12,

Guzmán, R.; Ancheyta, J.; Trejo, F. y Rodríguez, S. (2017). Methods for Determining Asphaltene Stability in Crude Oils. Fuel, 188, 530-543. doi: 10.1016/j.fuel.2016.10.012

Hasanvand, M. Z.; Montazeri, M.; Salehzadeh, M.; Amiri, M. y Fathinasab, M. (2018). A Literature Review of Asphaltene Entity, Precipitation, and Deposition: Introducing Recent Models of Deposition in the Well Column. Journal of Oil, Gas and Petrochemical Sciences, 1(3), 83-89. doi: org/10.30881/ jogps.00016

Kanehashi, S.; Masuda, R.; Yokoyama et al. (2015). Development of a Cashew Nut Shell Liquid (CNSL)based Polymer for Antibacterial Activity. Journal of Applied Polymer Science, 132(45), 4272542734. doi: $10.1002 /$ APP. 42725

Kraiwattanawong, K.; Fogler, H. S.; Gharfeh, S. G. et al. (2009). Effect of Asphaltene Dispersants on Aggregate Size Distribution and Growth. Energy \& Fuels, 23, 1575-1582. doi: 10.1021/ef800706c

Kuang, J.; Meléndez-Álvarez, A. A.; Yarbrough, J. et al. (2019). Assessment of the Performance of Asphaltene Inhibitors Using a Multi-Section Packed Bed Column. Fuel, 241, 247-254. doi: 10.1016/j. fuel.2018.11.059

Kusrini, E.; Mawarni, D. P.; Mamat, M.; Prasetyanto, E. A.; Usman, A. (2018). Comparison of Antibacterial Activity in Ethanol Extract and Essential Oil of Citrus sinensis (L.) Peels Obtained by Sohxlet and Distillation Methods. In The International Fundamentum Sciences Symposium, Terengganu. Malaysia. doi: 10.1088/1757-899X/440/1/012028

Li, H.; Zhang, J.; Xu, Q. et al. (2020). Influence of Asphaltene on Wax Deposition: Deposition Inhibition and Sloughing. Fuel, 266, 117047-117055. doi: 10.1016/j.fuel.2020.117047

Lim, S. H.; Go, K. S.; Kwon, E. H.; Nho, N. S., y Lee, J. G. (2020). Investigation of Asphaltene Dispersion Stability in Slurry-Phase Hydrocracking Reaction. Fuel, 271, 117509- 117517. doi: 10.1016/j.fuel.2020.117509

Mardani, E.; Mokhtari, B.y Soltani, B. (2018). Comparison of the Inhibitory Capacity of Vegetable Oils, and their Nonionic Surfactants on Iran Crude Oil Asphaltene Precipitation Using Quartz Crystal Microbalance. Petroleum Science and Technology, 36(11), 744-749. doi: 10.1080/10916466.2018.1445103

Marín, T., Marcano, S. y Febres, M. (2016). Evaluación del aceite de Jatropha curcas como aditivo dispersante de asfaltenos en un crudo del campo el Furrial, Venezuela. Ingeniería-Revista Académica de la Facultad de Ingeniería, 20(2), 98-107.

Marín, T. (2019). El aceite de coco (Cocos nucifera) como estabilizante de asfaltenos en un crudo del Estado Monagas, Venezuela: Efecto de la temperatura. Ingeniería y Desarrollo, 37(2), 290-305.

Martins, R. G.; Martins, L. S. y Santos, R. G. (2018). Effects of Short-Chain n-Alcohols on the Properties of Asphaltenes at Toluene/Air and Toluene/Water Interfaces. Colloids Interfaces, 2(13), 1-9. doi: 10.3390/colloids2020013

Meléndez-Álvarez, A. A.; Garcia-Bermudes, M.; Tavakkoli, M. et al. (2016). On the Evaluation of the Performance of Asphaltene Dispersants. Fuel, 179, 210-220. doi: 10.1016/j.fuel.2016.03.056

Nunes, M.; Yuan, L. L.; Weingart, D. et al. (2019). The Use of Cashew Nut Shell Liquid (CNSL) in PP/HIPS Blends: Morphological, Thermal, Mechanical and Rheological Properties. Materials, 12, 19041928. doi: $10.3390 / \mathrm{ma} 12121904$ 
Pereira, J. C.; Delgado-Linares, J.; Briones, A. et al. (2011). The Effect of Solvent Nature and Dispersant Performance on Asphaltene Precipitation from Diluted Solutions of Instable Crude Oil. Petroleum Science and Technology, 29(23), 2432-2440. doi: 10.1080/10916461003735061

Quiminet (2020). Precios de dispersantes de asfaltenos. Disponible en: http://bit.do/asphaltenesprice

Riss, H.; Carniel, T. K.; Farina, C. F. et al. (2015). Extração de óleo de chia (Salvia hispanica L.) via Sohxlet. In XI Congresso Brasileiro de Engenharia Química em Iniciação Científica. Campinas, Brasil.

Rodríguez, O.J. (2011). Evaluación de la productividad en el campo el Furrial posterior a las estimulaciones matriciales no reactivas (Tesis de pregrado). Caracas, Venezuela: Universidad Central.

Sánchez, L., Chávez, J., Ríos, L. A., y Cardona, S. M. (2015). Evaluación de un Antioxidante Natural extraído del Marañón (Anacardium occidentale L.) para mejorar la Estabilidad Oxidativa del Biodiesel de Jatropha. Información Tecnológica, 26(6), 19-30. doi: 10.4067/S0718-07642015000600004

Silva, F. B.; Guimarães, M.; Seidl, P. R., y García, M. (2013). Extraction and Characterization (compositional and thermal) of Asphaltenes from Brazilian Vacuum Residues. Brazilian Journal of Petroleum and Gas, 7(3), 107-118. doi: 10.5419/bjpg2013-0009

Taiwo, E. A. (2015). Cashew Nut Shell Oil - A Renewable and Reliable Petrochemical Feedstock. In Advances in Petrochemicals. doi: 10.5772/61096

Zeng, C.; Brunner, M.; Li, H.; Zhang, D. y Atkin, R. (2019). Dissolution and Suspension of Asphaltenes with Ionic Liquids. Fuel, 238, 129-138. doi: 10.1016/j.fuel.2018.10.070 